\title{
Pioglitazone counteracts the tumor necrosis factor-a inhibition of follicle-stimulating hormone-induced follicular development and estradiol production in an in vitro mouse preantral follicle culture system
}

\author{
Shuichiro Hara, Toshifumi Takahashi", Mitsuyoshi Amita, Koki Matsuo, Hideki Igarashi and Hirohisa Kurachi
}

\begin{abstract}
Background: Polycystic ovary syndrome (PCOS) is a common endocrine disorder in women of reproductive age and is characterized by chronic anovulation. Insulin resistance may be a key component of the pathogenesis of this disorder. Pioglitazone is a thiazolidinedione derivative that acts by improving insulin resistance via the peroxisome proliferator-activated receptor- $\gamma$ (PPAR- $\gamma$ ) pathway. Reportedly, pioglitazone improves the anovulation status in patients with PCOS. In the present study, we examined whether pioglitazone directly affects ovarian follicular development and steroidogenesis using in vitro mouse preantral follicle culture system.

Methods: An isolated individual in vitro mouse preantral follicle culture was used to test the effects of pioglitazone on the follicle development and steroidogenesis. Tumor necrosis factor-a (TNF-a), which plays a role in insulin resistance, has been reported to inhibit the follicle stimulating hormone (FSH)-induced follicular development and steroidogenesis in an in vitro mouse preantral follicle culture system. Therefore, we examined whether pioglitazone counteracts these effects by TNF-a. We assessed the follicle diameter and follicle survival and antral-like cavity formation rates, the $17 \beta$-estradiol (E2) levels in the culture medium, and the ovulation rate using the in vitro preantral follicle culture.

Results: Pioglitazone treatment counteracted the inhibition of TNF-a in FSH-induced follicle development in a dose-dependent manner. Pioglitazone, at a concentration of $5 \mu \mathrm{M}$, which was the minimum effective concentration, significantly counteracted the inhibition of TNF-a in FSH-induced follicle survival (29 versus 56\%, $P<0.05$ ), antral-like cavity formation ( 29 versus $48 \%, P<0.05$ ), E2 concentration in the culture medium (mean \pm $\mathrm{SEM}=21 \pm 1$ versus mean $\pm \mathrm{SEM}=27 \pm 1 \mathrm{pg} / \mathrm{mL}, P<0.05)$, and human chorionic gonadotropin-induced ovulation rate $(9$ versus $28 \%, P<0.05)$.

Conclusions: Pioglitazone counteracted the inhibition by TNF-a on FSH-induced follicle development and steroidogenesis in the in vitro mouse preantral follicle culture. The results suggest that pioglitazone may directly affect the follicular development and steroidogenesis.
\end{abstract}

Keywords: Polycystic ovary syndrome, Pioglitazone, TNF-a, Follicle culture, PPAR- $\gamma$

* Correspondence: totakaha@med.id.yamagata-u.ac.jp

Department of Obstetrics and Gynecology, Yamagata University Faculty of

Medicine, Yamagata 990-9585, Japan 


\section{Background}

Polycystic ovary syndrome (PCOS) is a common endocrine disorder in women of reproductive age, affecting $5-10 \%$ of the population [1], and is characterized by anovulation and infertility. Although the exact pathogenesis of PCOS remains unknown, insulin resistance and compensatory hyperinsulinemia are considered relevant [2]. Hyperinsulinemia contributes to excessive ovarian androgen production and the reduction of the hepatic synthesis of sex hormone-binding globulins, resulting in an elevated level of free androgen $[3,4]$. Intraovarian hyperandrogenism suppresses araomatase activity and $17 \beta$-estradiol (E2) production in granulosa cells $[5,6]$ and proliferation and arresting antral follicle development.

The circulating levels of adipocytokines, such as tumor necrosis factor- $\alpha$ (TNF- $\alpha$ ) and interleukin 6 , which raise insulin resistance, are higher in obese and nonobese women with PCOS than those in weight matched non-PCOS control women $[7,8]$. TNF- $\alpha$ is critcically involved in the apoptosis of preantral and antral follicles in mouse and human ovaries $[9,10]$. Moreover, TNF- $\alpha$ directly affects the ovarian steroidogenesis in granulosa cells in in vitro culture [11].

Insulin resistance with compensatory hyperinsulinemia is observed in approximately $70 \%$ of patients with PCOS, irrespective of obesity $[12,13]$. Several studies have suggested that decreases in insulin secretion improve infertility and ovulation [14-16]. Therefore, patients with PCOS are often treated with insulin-sensitizing drugs such as metformin and thiazolidinedione derivatives [17]. Pioglitazone is a thiazolidinedione derivative that has been used for the treatment of type 2 diabetes mellitus. Pioglitazone decreases peripheral insulin resistance via the peroxisome proliferator-activated receptor- $\gamma$ (PPAR- $\gamma$ ) pathway [18]. Several studies have demonstrated that pioglitazone improves clinical, hormonal, and metabolic status in patients with PCOS [15,19-21]. Moreover, pioglitazone directly inhibits estradiol and testosterone production in human ovarian cells in vitro [22]. Pioglitazone-stimulated reduction in peripheral insulin resistance and its direct effect on the ovaries might be effective for inducing ovulation in patients with PCOS. However, the direct effects of pioglitazone on the ovarian follicular development are still unclear.

We previously established the in vitro mouse preantral follicle culture system to assess the effects of bezafibrate, a lipid-lowering fibrate, on follicular development [23]. As bezafibrate is a nonselective ligand for PPAR- $\alpha, \delta$, and $\gamma[24,25]$, we demonstrated that the bezafibrate may exert direct action on the ovarian follicular development and steroidogenesis through the PPAR- $\gamma$ pathway [23]. Because pioglitazone is a specific PPAR $-\gamma$ agonist, we hypothesized that pioglitazone may also directly affect the ovarian follicular development and steroidogenesis.
The aim of this study was to examine whether pioglitazone affects the ovarian follicular development and steroidogenesis using an in vitro mouse preantral follicle culture system.

\section{Methods \\ Animals}

This study was performed with permission from the Committee of Animal Experimentation at the Yamagata University Faculty of Medicine. Mature female ICR mice at 6 weeks or older were used in this study. They were housed in a temperature- and light-controlled room at 23$25^{\circ} \mathrm{C}$, on a 12-h light:dark cycle (lights on, 0700-1900 h) and fed pellet food and water ad libitum.

\section{Reagents}

Human pituitary follicle-stimulating hormone (FSH) (cata$\log$ no. F4021) and TNF- $\alpha$ (catalog no. T6674), were purchased from Sigma-Aldrich ${ }^{\bullet}$ (St. Louis, MO). Pioglitazone was kindly donated by Takeda Pharmaceutical Company Limited, Osaka, Japan.

\section{Collection of preantral follicles}

Collection of preantral follicles from the mice was reported previously [23]. Briefly, female mice were killed by cervical dislocation, and early preantral follicles were mechanically isolated from the ovaries using a 30-gauge needle under a stereomicroscope. The collected preantral follicles were placed and pooled in N-2-hydroxyethylpiperazine-N'-2ethanesulfonic acid (HEPES)-minimal essential medium (MEM, catalog no. 12360038; Invitrogen Corp, Carlsbad, CA) supplemented with $5 \%$ fetal bovine serum (FBS; catalog no. 12603c; JRH bioscience, Lenexa, KS). After washing with HEPES-MEM 2 times, follicles with the following characteristics were selected: (1) diameter, 120-150 $\mu \mathrm{m}$; (2) immature oocyte centrally located within the follicle; (3) intact basal membrane; and (4) surrounded by theca cells [26]. These procedures were performed at $37^{\circ} \mathrm{C}$.

\section{In vitro preantral follicle culture}

In vitro preantral follicle culture was reported previously [23]. The follicle culture medium consisted of $\alpha$ MEM GlutaMax ${ }^{\text {TM }}$ (catalog no. 32561037; Invitrogen Corp., Carlsbad, CA) supplemented with 5\% FBS, 100 units $/ \mathrm{mL}$ penicillin, $100 \mu \mathrm{g} / \mathrm{mL}$ streptomycin, $5 \mu \mathrm{g} / \mathrm{mL}$ insulin, $5 \mu \mathrm{g} / \mathrm{mL}$ transferrin, and $5 \mathrm{ng} / \mathrm{mL}$ selenium (ITS; Invitrogen Corp., Carlsbad, CA). The medium was sterilized with a $20-\mu \mathrm{m}$ pore filter after the addition of supplements, which resulted in the basal medium. As $\mathrm{FSH}$ treatment at $100 \mathrm{mIU} / \mathrm{mL}$ was the minimum effective concentration for developing follicles (data not shown), $100 \mathrm{mIU} / \mathrm{mL}$ FSH were added to the basal medium in the in vitro follicle culture system. Follicles were individually cultured per well in a 24-well plate 
$\left(\right.$ Falcon $^{\mathrm{Tm}}$; Becton Dickinson and Company, Franklin Lakes, $\mathrm{NJ}$ ) containing $1 \mathrm{~mL}$ culture medium at $37^{\circ} \mathrm{C}$ with $5 \% \mathrm{CO}_{2}$ in air atmosphere. Follicles were cultured for 13 days. Subsequently, follicular growth was monitored every 2 days (day 2, 4, 6, 8, 10, and 12 of culture) under an inverted microscope at a $200 \times$ magnification. Each follicle image was captured by a cooled charge-coupled device camera system (DP-50; Olympus, Tokyo, Japan), and follicle diameter was measured using Image J imaging system software version 1.43 (National Institutes of Health, Bethesda, MD). A total of $500 \mu \mathrm{L}$ conditioned medium was refreshed with preincubated medium every 2 days (day 2, 4, 6, 8, 10, and 12). The spent media collected on the indicated days were pooled per culture plate and frozen at $-80^{\circ} \mathrm{C}$ for a successive E2 determination. When follicles clearly formed an antral-like cavity on day 12, ovulation and meiotic resumption were induced by refreshing media with culture media supplemented with $5 \mathrm{IU} / \mathrm{mL}$ human chorionic gonadotropin (hCG, Mochida Pharmaceutical Co., Tokyo, Japan). On day 13, follicles were checked for ovulation $16 \mathrm{~h}$ after hCG administration, and ovulation was considered when the follicle was visually ruptured and the cumulus oocyte complex (COC) extruded from the follicles. The ovulated COCs were evaluated for cumulus expansion and germinal vesicle breakdown (GVBD), indicating meiotic resumption of oocytes, after removing the surrounding cumulus cells by repeated pipetting in the presence of $300 \mathrm{IU} / \mathrm{mL}$ hyaluronidase.

\section{Assessment of follicle survival}

Using the method reported by Adriaenssens et al. [27] and Lenie et al. [28], we assessed follicle morphology and classified the follicular development stage as follicular, diffuse, or antral in the in vitro preantral follicle culture. Briefly, the follicular stage is characterized by preservation of the follicle basal membrane. Initially, theca cells grow out to the culture plate and granulosa cell proliferation is limited. The follicles remain in this stage until day 4 of culture. The diffuse stage is characterized by marked granulosa cell proliferation and a large preantral follicle. From day 4 to day 6 of culture, granulosa cells overgrow the theca cell monolayer through the basement membrane, and the follicles gradually enlarge. From day 6 or 8 up to day 12 of culture, granulosa cells differentiate into 2 spatially and functionally distinct populations, cumulus cells and mural granulosa cells, and the follicles form an antral-like cavity, which characterizes the antral stage.

In this culture system, surviving follicles were defined as those that could retain their oocyte completely embedded within the granulosa cell mass and that exhibited no signs of degeneration [28,29]. Follicle degeneration was characterized by failure of the granulosa cells to multiply, release oocytes, or by collapse [28].

\section{$17 \beta$-estradiol measurement}

Media cultured with individual mouse follicles were replaced every other day, and the collected media were pooled and stored at $-80^{\circ} \mathrm{C}$ until the measurement of E2 levels was performed. E2 levels in the collected culture media were measured using the estradiol enzyme immunoassay kit (Estradiol EIA Kit) (catalogue no. 582251; Cayman Chemical Co., Ann Arbor, MI) according to the manufacturer's protocol. Culture media from non-surviving follicles were excluded from the experiment.

\section{Experimental conditions and evaluation parameters}

In this experiment, the control group consisted of follicles cultured in media without FSH in the presence of the vehicle. All compounds were added to the culture media during the in vitro culture period. TNF- $\alpha$ was dissolved in distilled water at $10 \mu \mathrm{g} / \mathrm{mL}$ and was stored at $-80^{\circ} \mathrm{C}$ until use. Pioglitazone was dissolved in dimethyl sulfoxide (DMSO) at $10 \mathrm{mM}$ and was stocked at $-80^{\circ} \mathrm{C}$ until use. The final concentration of DMSO in the medium never exceeded $0.1 \%$. In the preliminary study, we tested the effects of DMSO as a vehicle on follicle development. There were no significant differences of follicle development between the groups treated with and without DMSO (data not shown).

Effects of various treatments on follicle development and steroidogenesis were evaluated as follows: follicle diameter, follicle survival, antral-like cavity formation, and measurement of E2 concentration in culture media. Moreover, we evaluated the effects of various treatments on ovulation and GVBD, indicating resumption of meiosis.

We previously demonstrated that TNF- $\alpha$ significantly inhibited FSH-induced follicular development and steroidogenesis in a dose-dependent manner [23]. In the present study, we examined the effects of pioglitazone on the follicle development and steroidogenesis by using the in vitro follicle culture system. Experimental scheme shows in Figure 1.

\section{Statistical analysis}

All experiments consisted of at least 5 independent experimental runs. Differences between means were calculated by one-way ANOVA, followed by a post hoc test, and data expressed as percentages were analyzed by Fisher's exact probability test using GraphPad Prism version 5.0 for Windows (GraphPad Software Inc., San Diego, CA). Values are given as mean \pm SEM. Significant differences are defined as $P<0.05$.

\section{Results}

Our previous data show that $100 \mathrm{mIU} / \mathrm{mL}$ of FSH treatment significantly induced the follicle growth and increase in E2 concentration in the culture medium compared to control samples without FSH treatment [23]. While follicles 


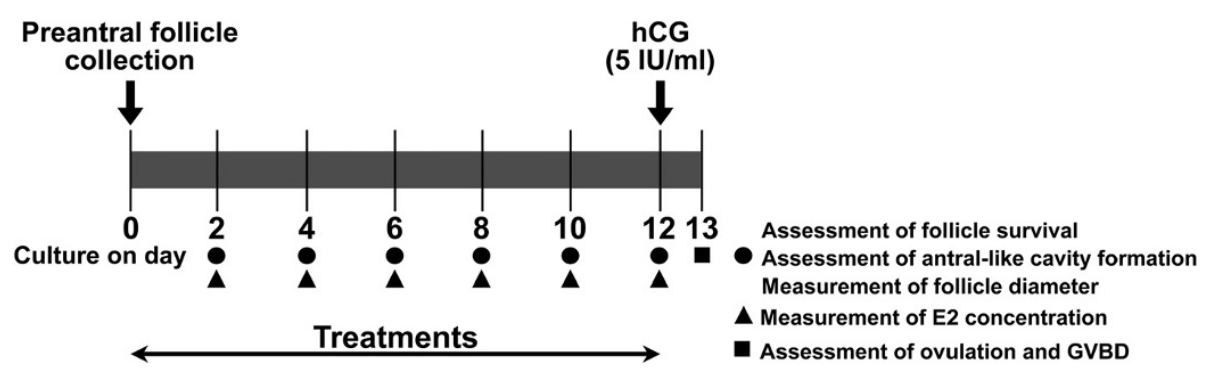

Figure 1 Study scheme. Scheme used for in vitro culture of mouse follicles for the assessment of follicle diameter, follicle survival, antral-like cavity formation, 17ß-estradiol (E2) concentration in the culture media, ovulation, and germinal vesicle breakdown (GVBD) after human chorionic gonadotropin (hCG)treatment is shown. Early preantral follicles were mechanically isolated from mice ovaries as described in Methods. Collected follicles were individually cultured per well in a 24-well plate for 12 days. Follicles were cultured with various treatments. The follicles were inspected for morphology, follicle survival, and antral-like cavity formation every other day as described in Methods. Media were refreshed every other day, and E2 concentrations in the collected media were measured. After 12 days of culture, $\mathrm{hCG}(5 \mathrm{IU} / \mathrm{mL})$ was added to the culture media to induce ovulation. After $16 \mathrm{~h}$ of hCG treatment, ovulation and GVBD were evaluated.

did not grow when cultured without FSH (Figure 2, upper panel), follicles cultured with FSH grew larger and formed an antral-like cavity at 10 to 12 days of culture (Figure 2, lower panel).

TNF- $\alpha$ is a pro-inflammatory cytokine that induces cell death [30]. TNF- $\alpha$ also influences follicle development and steroidogenesis [11]. Further, in patients with PCOS, serum and follicular fluid levels of TNF- $\alpha$ are higher than in patients without PCOS $[31,32]$. Therefore, we examined the effects of TNF- $\alpha$ on follicular development. In the in vitro culture, TNF- $\alpha$ significantly inhibited FSH-induced follicular development and steroidogenesis in a dose-dependent manner with a minimal effective dose of $5 \mathrm{ng} / \mathrm{mL}$. Therefore, we used the in vitro preantral follicle culture system with TNF- $\alpha$ as a model to study follicular development of PCOS.

We examined whether pioglitazone could counteract the inhibition of FSH-induced follicular development and steroidogenesis by TNF- $\alpha$. Pioglitazone significantly counteracted the inhibition of FSH-induced follicular development and steroidogenesis by TNF- $\alpha$ in a dose-dependent manner (Figure 3). TNF- $\alpha$ ( $5 \mathrm{ng} / \mathrm{mL}$ ) inhibited the FSH-induced follicle survival rate. Although $1 \mu \mathrm{M}$ of pioglitazone failed to show an effect, both 5 and $10 \mu \mathrm{M}$ of pioglitazone significantly counteracted the inhibition of FSH-induced follicle survival by TNF- $\alpha$ (Figure 3A). TNF- $\alpha$ inhibited the FSH-induced antral-like cavity formation rate. Although $1 \mu \mathrm{M}$ of pioglitazone failed to show an effect, both 5 and $10 \mu \mathrm{M}$ of pioglitazone significantly counteracted the inhibition of FSH-induced antral-like cavity formation rates by TNF- $\alpha$. TNF- $\alpha$ (5 $\mathrm{ng} / \mathrm{mL}$ ) inhibited the FSH-induced E2 production (Figure 3C). Although $1 \mu \mathrm{M}$ of pioglitazone failed to show an effect, both 5 and $10 \mu \mathrm{M}$ of pioglitazone counteracted the inhibition of FSH-induced E2 production by TNF- $\alpha$ (Figure 3C). While TNF- $\alpha$ significantly decreased FSHinduced follicle diameter at 12 days of culture, pioglitazone did not counteract the inhibition by TNF- $\alpha$ in follicle diameter at 12 days of culture (Figure 3D).

We examined the effects of pioglitazone on the hCGinduced ovulation and meiotic resumption of oocytes with or without FSH and TNF- $\alpha$ (Table 1). While TNF- $\alpha$ ( $5 \mathrm{ng} / \mathrm{mL}$ ) inhibited the hCG-induced ovulation rate in the FSH-treated group, pioglitazone $(5 \mu \mathrm{M})$ significantly

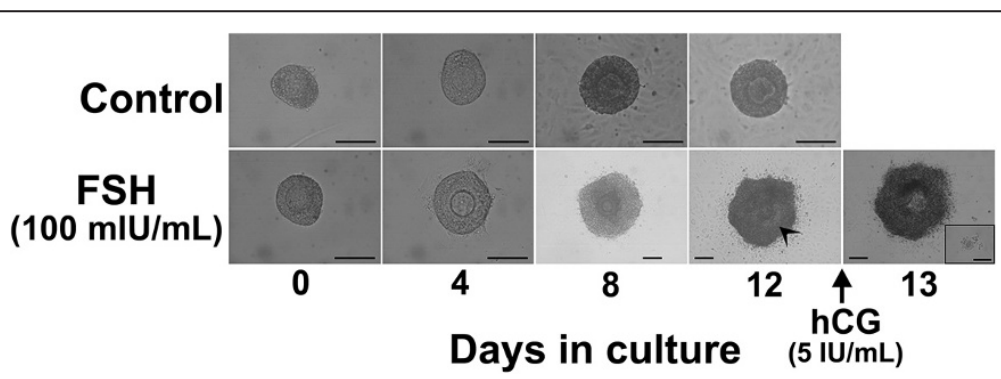

Figure 2 In vitro mouse preantral follicle culture system and the effects of FSH on follicular development. Examples of follicular development treated with or without follicle stimulating hormone $(\mathrm{FSH})(100 \mathrm{mlU} / \mathrm{mL})$ are shown. The majority of follicles not treated with FSH (control) grew in a multilayered pattern and did not survive. On the other hand, follicles cultured with FSH (100 mlU/mL) grew larger and formed antral-like cavities at 10 to 12 days of culture. The cumulus oocyte complex was visibly extruded from the antral-like cavity after $16 \mathrm{~h}$ of the administration of $5 \mathrm{IU} / \mathrm{mL}$ of hCG (inset). Bar $=100 \mu \mathrm{m}$. 
A

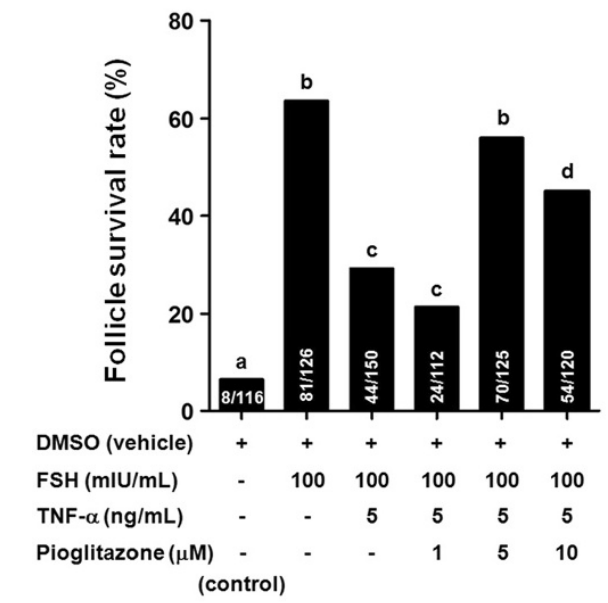

C

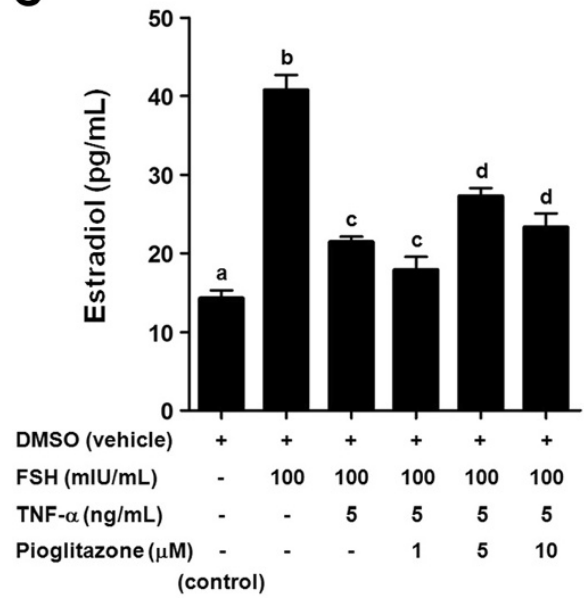

B

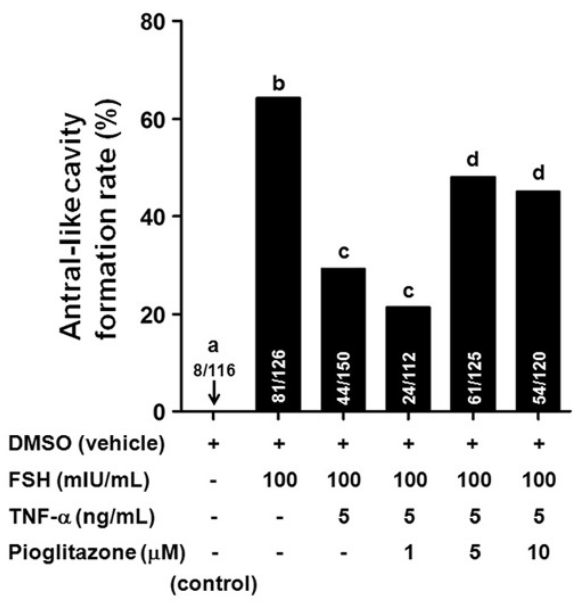

D

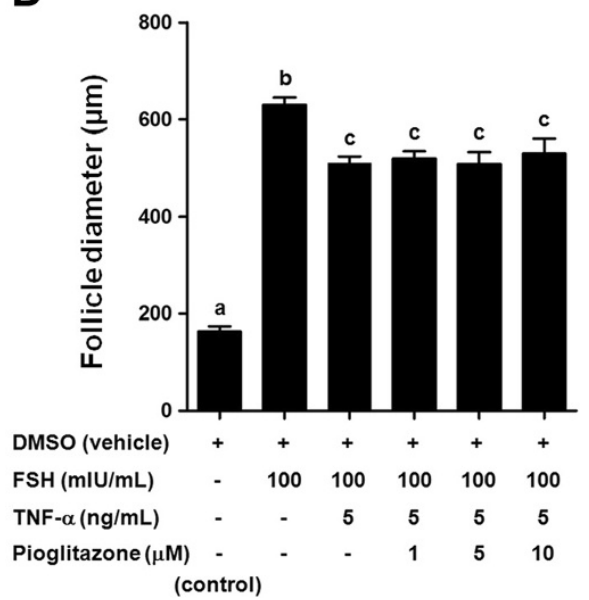

Figure 3 Pioglitazone counteracts the inhibition of FSH-induced follicular development and steroidogenesis by TNF-a. Effects of pioglitazone on the inhibition of follicle-stimulating hormone (FSH)-induced follicular development and steroidogenesis by tumor necrosis factora (TNF-a). Follicles were cultured with 1, 5, $10 \mu \mathrm{M}$ of pioglitazone in the presence or absence of $5 \mathrm{ng} / \mathrm{mL}$ of TNF-a or $100 \mathrm{mlU} / \mathrm{mL}$ of FSH. A) Follicle survival rates at 12 days of culture with various treatments are shown. Numbers inside bars indicate the number of surviving follicles/total tested follicles. B) Antral-like cavity formation rates at 12 days of culture with various treatments are shown. Numbers inside bars indicate the number of follicles with antral-like cavity/total tested follicles. C) $17 \beta$-estradiol (E2)concentrations in the culture media at 12 days of culture with various treatments are shown. D) Follicle diameters at 12 days of culture with various treatments are shown. Data are shown as the mean \pm SEM. The group treated only with dimethyl sulfoxide (DMSO) was used as a control. Bars with different letters represent a significant difference $(P<0.05)$.

Table 1 Effects of pioglitazone on ovulation and meiotic resumption in follicles treated with FSH and TNF-a

\begin{tabular}{|c|c|c|c|}
\hline Treatment & $\begin{array}{l}\text { Number of ovulated follicles/ } \\
\text { total tested follicles (\%) }\end{array}$ & $\begin{array}{l}\text { Number of ovulated follicles/number of follicles } \\
\text { with antral-like cavity formation (\%) }\end{array}$ & $\begin{array}{l}\text { Number of GVDB oocytes/ } \\
\text { number of ovulated COCs (\%) }\end{array}$ \\
\hline Control (DMSO) & $0 / 87(0)^{\mathrm{a}}$ & $0 / 0(0)^{\mathrm{a}}$ & $0 / 0(0)^{\mathrm{a}}$ \\
\hline TNF-a (5 ng/mL) & $0 / 72(0)^{a}$ & $0 / 0(0)^{a}$ & $0 / 0(0)^{\mathrm{a}}$ \\
\hline Pioglitazone $(5 \mu \mathrm{M})$ & $0 / 80(0)^{a}$ & $0 / 0(0)^{\mathrm{a}}$ & $0 / 0(0)^{a}$ \\
\hline $\mathrm{FSH}(100 \mathrm{mlU} / \mathrm{mL})$ & $60 / 126(48)^{b}$ & $60 / 81(74)^{b}$ & $60 / 60(100)^{b}$ \\
\hline $\mathrm{FSH}+\mathrm{TNF}-\mathrm{a}(5 \mathrm{ng} / \mathrm{mL})$ & $14 / 150(99)^{c}$ & $14 / 44(32)^{c}$ & $14 / 14(100)^{b}$ \\
\hline $\begin{array}{l}\text { FSH + TNF-a }(5 \mathrm{ng} / \mathrm{mL})+ \\
\text { pioglitazone }(5 \mu \mathrm{M})\end{array}$ & $35 / 125(28)^{d}$ & $35 / 60(58)^{d}$ & $35 / 35(100)^{b}$ \\
\hline
\end{tabular}

FSH, follicle stimulating hormone; TNF-a, tumor necrosis factor-a; DMSO, dimethyl sulfoxide; GVBD, germinal vesicle breakdown; COCs, cumulus oocyte complexes. a-d Values with different superscript letters within the same column differ significantly. $P<0.05$. 
counteracted the inhibition of the ovulation rates per total tested follicles and follicles with antral-like cavity formation by TNF- $\alpha$ in the FSH-treated groups. All oocytes underwent GVBD after COC ovulation in the FSH-treated groups.

\section{Discussion}

In the present study, we examined the direct effects of pioglitazone on the follicular development and steroidogenesis by using an in vitro mouse preantral follicle culture system. While TNF- $\alpha$ inhibited the FSH-induced follicle development, such as follicle survival, antral-like cavity formation, and E2 production, pioglitazone counteracted these inhibitory effects by TNF- $\alpha$.

In vitro mouse ovarian culture methods provide a useful tool for studying follicle development [23,33-35]. We employed an in vitro mouse ovarian follicle culture system for up to 12 days to study the effects of various compounds on FSH-induced follicle development and steroidogenesis. The mechanism of TNF- $\alpha$ inhibition of FSH-induced follicle development and steroidogenesis remains unknown. TNF- $\alpha$ is a well-known cytokine that is capable of inducing apoptosis in cells [30]. TNF- $\alpha$ is also known as adipocytokine that can induce insulin resistance [36]. In the ovary, TNF- $\alpha$ is critically involved in the follicles apoptosis $[9,10]$. Moreover, TNF- $\alpha$ has direct effects on ovarian steroidogenesis $[11,37]$. Several studies have reported that TNF- $\alpha$ decreased steroidogenic acute regulatory protein (StAR) mRNA and protein levels [38,39].

In the present study, we showed that pioglitazone counteracted the TNF- $\alpha$ inhibition in FSH-induced follicle development and steroidogenesis in an in vitro culture system. The precise mechanisms of restoration of TNF- $\alpha$ inhibition of FSH-induced follicle development and steroidogenesis by pioglitazone were unknown. Recently, we examined whether bezafibrate, a ligand for PPARs $(\alpha, \delta, \gamma)$ rescued the TNF- $\alpha$ inhibition of follicular development and steroidogenesis [23]. Our previous data showed that PPAR- $\gamma$ was expressed in mouse preantral follicles and that GW1929, a selective PPAR- $\gamma$ agonist, counteracted the inhibition of FSH-induced follicular development by TNF- $\alpha$. Furthermore, GW9662, a selective PPAR- $\gamma$ antagonist, canceled the restorative effects of bezafibrate on the inhibition of FSH-induced follicular development and steroidogenesis by TNF- $\alpha$. Therefore, we suggested that, among the three PPARs, PPAR- $\gamma$ is important for follicular development. In fact, it is reported that deletion of PPAR- $\gamma$ results in impaired fertility in genetically targeted mice [40].

It is well known that thiazolidinediones, such as pioglitazone and rosiglitazone, specifically bind to PPAR- $\gamma$, which is located on macrophages, adipose tissue or human granulosa lutein cells [18,41]. Pioglitazone is a thiazolidinedione derivative that acts by improving insulin resistance via the PPAR- $\gamma$ pathway. Pioglitazone improves peripheral insulin resistance, ovulation, and hyperandrogenism in women with PCOS [42-44]. SetoYoung et al. reported that pioglitazone has a direct action on the human ovary [22]. Kim et al. showed that pioglitazone decreased the follicular fluid levels of TNF- $\alpha$ in patients with PCOS [45]. Moreover, several reports have demonstrated that PPAR- $\gamma$ activation reduced the expression of TNF- $\alpha$ and other inflammatory cytokines in vivo $[46,47]$. Pioglitazone may act on PPAR- $\gamma$ and reduce TNF- $\alpha$. However, the detailed pathway through which pioglitazone suppresses TNF- $\alpha$ is unclear. Further examinations are needed to determine the detailed mechanism by which pioglitazone affects follicular development.

\section{Conclusions}

Pioglitazone counteracted the inhibition of FSH-induced follicular development and steroidogenesis by TNF- $\alpha$. These results suggest that pioglitazone may directly act on ovarian functions through PPAR- $\gamma$ activation in patients with PCOS.

\section{Abbreviations}

PCOS: Polycystic ovary syndrome; PPAR- - : Peroxisome proliferator-activated receptor- $\gamma$; TNF-a: Tumor necrosis factor-a; FSH: Follicle stimulating hormone; E2: 17ß-estradiol; hCG: Human chorionic gonadotropin; HEPES: N-2-

hydroxyethylpiperazine-N'-2-ethanesulfonic acid; MEM: Minimal essential medium; COC: Cumulus oocyte complex; GVBD: Germinal vesicle breakdown; DMSO: Dimethyl sulfoxide.

\section{Competing interest}

The authors declare that they have no competing interests.

\section{Authors' contributions}

$\mathrm{SH}$ carried out the experiment of follicle culture and drafted the manuscript. TT participated in the design of the study and drafted the manuscript. MA carried out the measurement of E2. HI performed the statistical analysis. HK participated in its design and coordination and helped to draft the manuscript. All authors read and approved the final manuscript.

\section{Acknowledgements}

Grant support: This study was supported by a Grant-in-aid for General Science Research No. 22591815 to Toshifumi Takahashi, 22390308 and 24659723 to Hirohisa Kurachi, and the Global COE Program for Medical Sciences from the Japan Society for the Promotion of Science.

Received: 12 August 2013 Accepted: 17 September 2013 Published: 30 September 2013

\section{References}

1. Asuncion M, Calvo RM, San Millan JL, Sancho J, Avila S, Escobar-Morreale HF: A prospective study of the prevalence of the polycystic ovary syndrome in unselected Caucasian women from Spain. J Clin Endocrinol Metab 2000, 85:2434-2438.

2. American College of Obstetricians and Gynecologists: ACOG practice bulletin no. 108: polycystic ovary syndrome. Obstet Gynecol 2009, 114:936-949.

3. Nestler JE: Metformin for the treatment of the polycystic ovary syndrome. N Engl J Med 2008, 358:47-54.

4. Amin M, Abdel-Kareem O, Takekida S, Moriyama T, Abd el-Aal G, Maruo T: Minireview: up-date management of non responder to clomiphene citrate in polycystic ovary syndrome. Kobe J Med Sci 2003, 49:59-73.

5. Agarwal SK, Judd HL, Magoffin DA: A mechanism for the suppression of estrogen production in polycystic ovary syndrome. J Clin Endocrinol Metab 1996, 81:3686-3691. 
6. Jakimiuk AJ, Weitsman SR, Brzechffa PR, Magoffin DA: Aromatase mRNA expression in individual follicles from polycystic ovaries. Mol Hum Reprod 1998, 4:1-8.

7. Gonzalez F, Thusu K, Abdel-Rahman E, Prabhala A, Tomani M, Dandona P: Elevated serum levels of tumor necrosis factor alpha in normalweight women with polycystic ovary syndrome. Metabolism 1999, 48:437-441.

8. Tarkun I, Cetinarslan B, Turemen E, Canturk Z, Biyikli M: Association between circulating tumor necrosis factor-alpha, interleukin-6, and insulin resistance in normal-weight women with polycystic ovary syndrome. Metab Syndr Relat Disord 2006, 4:122-128.

9. Morrison LJ, Marcinkiewicz JL: Tumor necrosis factor alpha enhances oocyte/follicle apoptosis in the neonatal rat ovary. Biol Reprod 2002, 66:450-457.

10. Hussein MR: Apoptosis in the ovary: molecular mechanisms. Hum Reprod Update 2005, 11:162-177.

11. Basini G, Mainardi GL, Bussolati S, Tamanini C: Steroidogenesis, proliferation and apoptosis in bovine granulosa cells: role of tumour necrosis factor-alpha and its possible signalling mechanisms. Reprod Fertil Dev 2002, 14:141-150.

12. DeUgarte CM, Bartolucci AA, Azziz R: Prevalence of insulin resistance in the polycystic ovary syndrome using the homeostasis model assessment. Fertil Steril 2005, 83:1454-1460.

13. Diamanti-Kandarakis $E$, Dunaif A: Insulin resistance and the polycystic ovary syndrome revisited: an update on mechanisms and implications. Endocr Rev 2012, 33:981-1030.

14. Mathur R, Alexander CJ, Yano J, Trivax B, Azziz R: Use of metformin in polycystic ovary syndrome. Am J Obstet Gynecol 2008, 199:596-609.

15. Kim CH, Jeon GH, Kim SR, Kim SH, Chae HD, Kang BM: Effects of pioglitazone on ovarian stromal blood flow, ovarian stimulation, and in vitro fertilization outcome in patients with polycystic ovary syndrome. Fertil Steril 2010, 94:236-241.

16. Iuorno MJ, Nestler JE: Insulin-lowering drugs in polycystic ovary syndrome. Obstet Gynecol Clin North Am 2001, 28:153-164

17. Tang T, Lord JM, Norman RJ, Yasmin E, Balen AH: Insulin-sensitising drugs (metformin, rosiglitazone, pioglitazone, D-chiro-inositol) for women with polycystic ovary syndrome, oligo amenorrhoea and subfertility. Cochrane Database Syst Rev 2012, 5:CD003053.

18. Cariou B, Charbonnel B, Staels B: Thiazolidinediones and PPARgamma agonists: time for a reassessment. Trends Endocrinol Metab 2012, 23:205-215

19. Du Q, Yang S, Wang YJ, Wu B, Zhao YY, Fan B: Effects of thiazolidinediones on polycystic ovary syndrome: a meta-analysis of randomized placebocontrolled trials. Adv Ther 2012, 29:763-774.

20. Koo YA, Shin SY, Yoon BK, Choi D: Pioglitazone for treating polycystic ovary syndrome in non-obese women of reproductive age with different clinical presentations. Gynecol Endocrinol 2007, 23:461-467.

21. Ota H, Goto T, Yoshioka T, Ohyama N: Successful pregnancies treated with pioglitazone in infertile patients with polycystic ovary syndrome. Fertil Steril 2008, 90:709-713.

22. Seto-Young D, Paliou M, Schlosser J, Avtanski D, Park A, Patel P, Holcomb K, Chang P, Poretsky L: Direct thiazolidinedione action in the human ovary: insulin-independent and insulin-sensitizing effects on steroidogenesis and insulin-like growth factor binding protein-1 production. J Clin Endocrinol Metab 2005, 90:6099-6105.

23. Hara S, Takahashi T, Amita M, Igarashi H, Tsutsumi S, Kurachi H: Bezafibrate restores the inhibition of FSH-induced follicular development and steroidogenesis by tumor necrosis factor-alpha through peroxisome proliferator-activated receptor-gamma pathway in an in vitro mouse preantral follicle culture. Biol Reprod 2011, 85:895-906.

24. Tenenbaum A, Fisman EZ, Boyko V, Benderly M, Tanne D, Haim M, Matas Z Motro M, Behar S: Attenuation of progression of insulin resistance in patients with coronary artery disease by bezafibrate. Arch Intern Med 2006, 166:737-741

25. Tenenbaum A, Motro M, Fisman EZ, Schwammenthal E, Adler $Y$, Goldenberg I, Leor J, Boyko V, Mandelzweig L, Behar S: Peroxisome proliferator-activated receptor ligand bezafibrate for prevention of type 2 diabetes mellitus in patients with coronary artery disease. Circulation 2004, 109:2197-2202.
26. Lenie S, Smitz J: Functional AR signaling is evident in an in vitro mouse follicle culture bioassay that encompasses most stages of folliculogenesis. Biol Reprod 2009, 80:685-695.

27. Adriaenssens T, Mazoyer C, Segers I, Wathlet S, Smitz J: Differences in collagen expression in cumulus cells after exposure to highly purified menotropin or recombinant follicle-stimulating hormone in a mouse follicle culture model. Biol Reprod 2009, 80:1015-1025.

28. Lenie S, Cortvrindt R, Adriaenssens T, Smitz J: A reproducible two-step culture system for isolated primary mouse ovarian follicles as single functional units. Biol Reprod 2004, 71:1730-1738.

29. Adriaens I, Cortvrindt R, Smitz J: Differential FSH exposure in preantral follicle culture has marked effects on folliculogenesis and oocyte developmental competence. Hum Reprod 2004, 19:398-408.

30. Cleveland $J \mathrm{~L}$, Ihle JN: Contenders in FasL/TNF death signaling. Cell 1995, 81:479-482.

31. Amato G, Conte M, Mazziotti G, Lalli E, Vitolo G, Tucker AT, Bellastella A, Carella C, Izzo A: Serum and follicular fluid cytokines in polycystic ovary syndrome during stimulated cycles. Obstet Gynecol 2003, 101:1177-1182

32. Choi YS, Yang HI, Cho S, Jung JA, Jeon YE, Kim HY, Seo SK, Lee BS: Serum asymmetric dimethylarginine, apelin, and tumor necrosis factor-alpha levels in non-obese women with polycystic ovary syndrome. Steroids 2012, 77:1352-1358

33. Nayudu PL, Osborn SM: Factors influencing the rate of preantral and antral growth of mouse ovarian follicles in vitro. J Reprod Fertil 1992, 95:349-362.

34. Hartshorne GM: In vitro culture of ovarian follicles. Rev Reprod 1997, 2:94-104

35. Cortvrindt RG, Smitz JE: Follicle culture in reproductive toxicology: a tool for in-vitro testing of ovarian function? Hum Reprod Update 2002, 8:243-254.

36. Gonzalez F: Inflammation in polycystic ovary syndrome: underpinning of insulin resistance and ovarian dysfunction. Steroids 2012, 77:300-305.

37. Montgomery Rice V, Limback SD, Roby KF, Terranova PF: Tumor necrosis factor alpha inhibition of follicle-stimulating hormone-induced granulosa cell estradiol secretion in the human does not involve reduction of cAMP secretion but inhibition at post-cAMP site(s). Endocrine 1999, 10:19-23.

38. Abdo M, Hisheh S, Arfuso F, Dharmarajan A: The expression of tumor necrosis factor-alpha, its receptors and steroidogenic acute regulatory protein during corpus luteum regression. Reprod Biol Endocrinol 2008, 6:50,

39. Ma Y, Ren S, Pandak WM, Li X, Ning Y, Lu C, Zhao F, Yin L: The effects of inflammatory cytokines on steroidogenic acute regulatory protein expression in macrophages. Inflamm Res 2007, 56:495-501.

40. Cui Y, Miyoshi K, Claudio E, Siebenlist UK, Gonzalez FJ, Flaws J, Wagner KU, Hennighausen L: Loss of the peroxisome proliferation-activated receptor gamma (PPARgamma) does not affect mammary development and propensity for tumor formation but leads to reduced fertility. J Biol Chem 2002, 277:17830-17835.

41. Chen Q, Sun X, Chen J, Cheng L, Wang J, Wang Y, Sun Z: Direct rosiglitazone action on steroidogenesis and proinflammatory factor production in human granulosa-lutein cells. Reprod Biol Endocrinol 2009, 7:147.

42. Li XJ, Yu YX, Liu CQ, Zhang W, Zhang HJ, Yan B, Wang LY, Yang SY, Zhang $\mathrm{SH}$ : Metformin vs thiazolidinediones for treatment of clinical, hormonal and metabolic characteristics of polycystic ovary syndrome: a metaanalysis. Clin Endocrinol (Oxf) 2011, 74:332-339.

43. Aroda VR, Ciaraldi TP, Burke P, Mudaliar S, Clopton P, Phillips S, Chang RJ, Henry RR: Metabolic and hormonal changes induced by pioglitazone in polycystic ovary syndrome: a randomized, placebo-controlled clinical trial. J Clin Endocrinol Metab 2009, 94:469-476.

44. Brettenthaler N, De Geyter C, Huber PR, Keller U: Effect of the insulin sensitizer pioglitazone on insulin resistance, hyperandrogenism, and ovulatory dysfunction in women with polycystic ovary syndrome. J Clin Endocrinol Metab 2004, 89:3835-3840.

45. Kim CH, Ahn JW, You RM, Kim SH, Chae HD, Kang BM: Pioglitazone treatment decreases follicular fluid levels of tumor necrosis factor-alpha and interleukin-6 in patients with polycystic ovary syndrome. Clin Exp Reprod Med 2011, 38:98-102.

46. Ohama Y, Harada T, Iwabe T, Taniguchi F, Takenaka Y, Terakawa N Peroxisome proliferator-activated receptor-gamma ligand reduced 
tumor necrosis factor-alpha-induced interleukin-8 production and growth in endometriotic stromal cells. Fertil Steril 2008, 89:311-317.

47. Sharma AM, Staels B: Review: peroxisome proliferator-activated receptor gamma and adipose tissue-understanding obesity-related changes in regulation of lipid and glucose metabolism. J Clin Endocrinol Metab 2007, 92:386-395

doi:10.1186/1757-2215-6-69

Cite this article as: Hara et al:: Pioglitazone counteracts the tumor necrosis factor- $a$ inhibition of follicle-stimulating hormone-induced follicular development and estradiol production in an in vitro mouse preantral follicle culture system. Journal of Ovarian Research 2013 6:69.

\section{Submit your next manuscript to BioMed Central and take full advantage of:}

- Convenient online submission

- Thorough peer review

- No space constraints or color figure charges

- Immediate publication on acceptance

- Inclusion in PubMed, CAS, Scopus and Google Scholar

- Research which is freely available for redistribution 